\title{
Design and performance of an eight pole resistive magnet for soft x-ray magnetic dichroism measurements
}

\author{
Elke Arenholz \\ Advanced Light Source, Lawrence Berkeley National Laboratory, Berkeley, CA 94720
}

Soren O. Prestemon

Engineering Division, Lawrence Berkeley National Laboratory, Berkeley, CA 94720

\begin{abstract}
To take full advantage of the strengths of soft $\mathrm{x}$-ray magnetic dichroism (XMD) measurements for the detailed and quantitative characterization of multi-element magnetic materials, we developed an eight pole electromagnet that provides magnetic fields up to $0.9 \mathrm{~T}$ in any direction relative to the incoming $\mathrm{x}$-ray beam. The setup allows us to measure magnetic circular and linear dichroism spectra as well as to thoroughly study magnetization reversal processes with very high precision. Design constraints and system optimization for maximum peak field are discussed. The predicted current-field relation is in excellent agreement with experimental findings. A brief discussion of the key technical difficulties in developing a similar superconducting device with peak fields of $5 \mathrm{~T}$ and ramping rates suitable for point-by-point full field reversal in an XMD experiment is presented.
\end{abstract}




\section{SCIENTIFIC MOTIVATION}

Since its theoretical prediction and first observation in the 1980s [1-4], x-ray magnetic dichroism (XMD) in absorption, reflection, scattering, fluorescence etc. has contributed enormously to our understanding of magnetic materials. It is unique in its intrinsic element-specificity [5] and chemical-site sensitivity [6] that allows separating the contributions of multiple magnetic species in alloys or layered systems [7]. The importance of soft $\mathrm{x}$-rays for XMD experiments arises from the fact that the transition metal $(\mathrm{Fe}, \mathrm{Co}, \mathrm{Ni}, \ldots) \mathrm{L}$ edges and the rare earth $(\mathrm{Gd}, \mathrm{Tb}, \mathrm{Dy}, \ldots) \mathrm{M}$ edges fall into the 500 to $1500 \mathrm{eV}$ range. Through the dipole allowed $2 p \rightarrow 3 d$ and $3 d \rightarrow 4 f$ transitions they probe the $3 d$ and $4 f$ valence states that determine largely the magnetic properties of transition metals and rare earth, respectively. Most importantly, theoretically derived "sum rules" link $\mathrm{x}$-ray magnetic circular dichroism (XMCD) intensities to spin and orbital magnetic moments [8-10] and x-ray magnetic linear dichroism (XMLD) signals to magnetocrystalline anisotropy energies $[11,12]$, i.e. sum rules make the use of $x$-rays for quantitative magnetometry possible.

To take full advantage of the strengths of XMD measurements for the detailed and quantitative characterization of complex multi-component magnetic materials the ability to apply magnetic fields at variable angles to the incoming $\mathrm{x}$-ray beam is essential. $\mathrm{XMCD}$ probes the magnetic component along the propagation direction of the $\mathrm{x}$-rays, i.e. the magnetic field is typically applied parallel to the incoming x-ray beam. XMLD spectra from ferri- or ferromagnetic materials are obtained with linearly polarized radiation by applying external fields parallel and perpendicular to the polarization direction in the plane perpendicular to the $\mathrm{x}$-ray beam. Consequently, to gain access to 
all information that XMCD and XMLD measurements provide, a magnet endstation should allow external fields along those principle directions (along the x-ray beam and along two orthogonal directions within the plane perpendicular to the photon beam). A device that provides magnetic fields along three orthogonal directions is capable of generating fields in any arbitrary direction by an appropriate superposition of the orthogonal components.

In addition to obtaining spectroscopic information from XMCD and XMLD data, arbitrary field directions also allow the thorough study of magnetization reversal in ferromagnetic systems [13]. Orthogonal magnetization components can be obtained by monitoring the field dependence of the XMCD signal for normal incidence (out-of-plane component) and two grazing incidence geometries with the sample rotated by $90^{\circ}$ around the sample normal (two orthogonal in-plane magnetization components) while keeping the relative orientation of external field and sample constant.

Different detection methods provide complementary information, e.g. electron yield measurements are surface sensitive while fluorescence yield and transmission experiments can probe the bulk of a sample. Consequently, a magnet endstation should be able to accommodate a series of different detection schemes (electron yield, transmission, fluorescence yield); access regions for detectors like diodes must therefore be available. The magnetic field in these regions has to be sufficiently small so as to not effect detector and signal.

For the application of XMD sum rules, spectra of very high precision are essential. They can be most easily obtained by reversing the applied magnetic field for each photon energy in an x-ray absorption spectrum, thus eliminating the influence of slow drifts of 
the photon source and of the limited repeatability in positioning optical elements in a monochromator [14].

A permanent magnet device [15] and a superconducting eight pole magnet [16] that provide magnetic fields in variable directions have been proposed and built in the past. However, to reverse the field in these devices takes typically tens of seconds to tens of minutes making the measurement of XMD spectra by point-by-point field reversal impractical.

Based on these considerations, we have developed an eight pole electromagnet that provides magnetic fields up to $0.9 \mathrm{~T}$ in any direction relative to the incoming $\mathrm{x}$-ray beam. Full field reversal can be achieved in $0.5 \mathrm{~s}$ suitable for point-by-point reversal in spectroscopy experiments. Total electron yield measurements are feasible by monitoring the sample drain current, as are total fluorescence yield and transmission experiments. In this article we describe the design, construction, and performance of the device.

\section{DESIGN CONSIDERATIONS AND OPTIMIZATION}

In addition to the basic capabilities of the magnet (variable field directions, field ramping rates sufficient for point-by-point field reversal in an XMD experiment, flexible sample/detector geometries) the following considerations determined the design of the system:

- The magnet was designed to be located completely outside the vacuum system, i.e. it surrounds a small vacuum chamber. This reduced the complexity of the system development vastly since none of the magnet components had to be vacuum compatible. 
The use of fluid feed-throughs for water cooling of the magnet coils and high power electrical feed-throughs became unnecessary reducing the risk of catastrophic vacuum failures. It also allowed standard epoxies to be used in the magnet fabrication.

- Three dipole pairs are sufficient to create arbitrary field orientations by superposition of the individual fields. However, an eight pole configuration makes it easier to achieve the desired field homogeneity and requires fewer Amp-turns per pole for the same field as a six pole configuration, thereby generating higher field. Furthermore, the geometry provides easier accessibility. The eight magnet poles are arranged at the corners of a cube. The vacuum chamber is in essence a six way cross oriented with the individual ports normal to the faces of this cube. The cube face-normals are referred to as "principle axes", i.e. $\mathbf{x}, \mathbf{y}, \mathbf{z}$ in this publication. While it may be possible to increase the field strength along some directions by assuming a less symmetric setup this configuration simplifies the current field-relationship significantly. To reduce fabrication cost and facilitate maintenance the eight coil pack/ pole units are identical.

- The minimum field increment is limited by the power-supply current step size. It also reduces the remanent field of the device at zero-current. Changing pole material from high permeability steel to non-magnetic Aluminum results in a decrease in the minimum field step size by almost one order of magnitude. To allow the change between magnetic steel and Aluminum poles for maximum field and highest field precisions around zero-field, respectively, the magnet coils are not wound directly on the poles but on $\mathrm{Al}$ mandrels, which are then mounted to the pole pieces.

- The system was built primarily for use at beamline 4.0.2 at the Advanced Light Source [17]. The horizontal beam sizes at the sample is $0.8 \mathrm{~mm}$, resulting in a $5 \mathrm{~mm}$ spot for $\mathrm{x}-$ 
ray beams at $10^{\circ}$ grazing incidence. The magnet was design for field uniformity of better than $1 \%$ over the area sampled by the $\mathrm{x}$-ray beam in this geometry.

- Access openings with 0.8 inches diameter to the center of the magnet were determined to be sufficient to allow the installation of a cryogenic sample holder with azimuthal rotation capability.

- The system is used mainly for three applications, i.e. constant field for the duration of a X-ray absorption scan, point-by-point field reversal for an XMD photon energy scan, and hysteresis loop measurements. The maximum field that can be reached in each case is limited by the ability to remove the power dissipated in the magnet coils by water cooling. The theoretical and experimental optimization of the water cooling was therefore an important aspect of the design.

The dimensions of magnet yoke and poles (taper, diameter, length) were optimized for maximum peak field in the magnet center using the software package TOSCA (by Vector Fields, Inc., Oxford, UK). General design conclusions can be made:

- The optimal taper angle is a function of the pole-tip diameter and the pole-to-pole gap, and should be chosen sufficiently large that saturation begins at the pole tip and not at the pole/yoke interface to maximize the central field, but should be minimized to maximize the amount of space for current-carrying conductor.

- The pole-tip diameter should also be optimized. A larger diameter allows for a higher field in the pole's unsaturated regime due to the enhanced scalar potential surface. However, in this regime some flux will pass through neighboring poles rather than through the central region; as the pole-tip diameter is increased, a larger fraction of 
the flux is diverted through the other poles. The result is ultimately a reduction in the center field. Optimization calculations demonstrate the importance of positioning some conductor in the pole tip region.

- The space between coil and pole (necessary to accommodate the aluminum mandrel) reduces the total current-carrying cross section and should be minimized.

- Increasing the pole length while keeping the number of Amp-turns constant does not have a strong impact on the peak field as long as the poles are not fully saturated; the length can therefore be optimized to provide sufficient cooling performance.

Based on the previous considerations, the design parameters for our magnet system result in the following system characteristics:

- Pole tips are fully saturated at coil currents of about $80 \mathrm{~A}$, and the current field relation starts to deviate from a linear relation around $50 \mathrm{~A}$.

- The stored energy in the system is $260 \mathrm{~J}$ when all poles are fully energized. The $L / R$ time constant of the system is about $0.1 \mathrm{~s}$ and its inductance $0.3 \mathrm{H}$. The calculated eddy-currents in the Al mandrel and in the pole decay on a similar time scale. The system can therefore be switched from one field vector to another within $0.5 \mathrm{~s}$ without further temporal field variations from decaying eddy currents.

- Changing the pole material to Holmium, which fully saturates at about $4 \mathrm{~T}$, will extend the range of linear current-field relationships but not lead to significant increase in the peak field $(\leq 10 \%)$ due to its lower permeability at low field. Since the pole material is interchangeable, this is still a possible upgrade. 
- The hoop stress in a coil pack is small $(<0.25 \mathrm{MPa})$ and easily supported by the monolithic Al mandrel/epoxy/coil structure. The attractive forces between the coils are less than $550 \mathrm{~N}$ per coil and are supported by the Al mandrel design. The force on a pole is about $675 \mathrm{~N}$ and is supported by the yoke structure.

- The weight of the system is about $320 \mathrm{lbs}(145 \mathrm{~kg})$.

\section{CONSTRUCTION}

The magnet consists of multiple components such as coil packs, magnet poles, yoke, vacuum chamber, support structure etc. and optimized sample holders are required for various applications of the system. In this section, we consider the fabrication of these components separately, although in reality the design and construction of the individual components is clearly closely linked.

\section{A. Coil pack}

We used $\mathrm{Cu}$ wire with a square cross section (0.076 inches by 0.076 inches) and an insulation thickness of 0.0008 inches for the coils. The low turn-to-turn voltage allows minimal insulation, i.e. insulation type and thickness were determined by mechanical strength and not dielectric strength. The coils are wet wound [18] on cone-shaped, anodized Aluminum mandrels that provide mechanical support and allow for the exchange of pole materials. The wire is wound in four layers; the first two layers start near the tip whereas the third and forth layer begin about 1/5 of the mandrel length away from the tip to accommodate the space constraints. All electrical terminals are located at 
the base of the coil pack. Each coil consists of a total of 180 turns in series. The coils are covered with three parallel cooling circuits (see Figure 1(a)): 2/5 of the coil near the tip with two independent circuits (1/16 inch OD, 0.0345 inch ID, 0.2 1/min flow) and an additional circuit for the lower part of the coil (3/16 inch OD, 0.1275 inch ID, $2.65 \mathrm{l} / \mathrm{min}$ flow). The hydraulic circuits emanate from a manifold located at, but isolated from, the electrical bus-bars. In addition, the poles are cooled internally by a squirt tube, i.e. two nested copper tubes providing supply and return path for cooling water are inserted into a cylindrical hole in the pole extending to within 1 inch of the pole tip (ID $1 / 2 \mathrm{inch}$ ). The cooling circuits covering the coils and the internal cooling of the poles provided by the squirt tube contribute about equally to removing the power dissipated in the system.

\section{B. Poles}

One set of magnet poles and the magnet yoke are made out of 1010 steel; a second set of Aluminum poles are used for low field applications to provide users with smaller incremental field steps. The taper of the poles was carefully matched to that of the mandrel. Thermal grease (Dow Corning ${ }^{\odot} 340$ ) was used to improve the thermal contact at the interface. A groove cut in the pole allows the installation of a thermocouple near the tip of the pole where tests showed the temperature rise is most significant during operation. The pole temperature is used for an interlock to the magnet power supplies to avoid overheating of the system. One of the eight coil pack/pole units is show in Figure 1(a). 


\section{Yoke}

Magnetic flux closure is achieved through a steel box yoke that holds all eight poles. The shared yoke does not significantly affect the magnet performance but simplifies the design. The yoke consists of two identical halves that surround the vacuum chamber, its eight faces are each normal to fictitious lines connecting opposite corners of a cube, i.e. the yoke is shaped like an octahedron. The faces determine the final location and angles of the poles and are toleranced accordingly. Part of the yoke is shown in Figures 1(b) and (c).

\section{Vacuum chamber}

The magnet surrounds a small non-magnetic Ti-alloy chamber. A center sphere of 2 inches diameter surrounds the magnetic center. Six access ports aligned at orthogonal axes are used for 1) the incoming X-ray beam, 2) a diode for transmission measurements, 3) pumping, 4) to insert the sample holder from the top, 5) a diode close to the sample at $90^{\circ}$ to the incoming $\mathrm{x}$-ray beam for total fluorescence yield measurements and 6) a viewport.

The base pressure of the system is typically $\leq 5 \cdot 10^{-8}$ Torr and can be improved to $2 \cdot 10^{-9}$ Torr after bake-out. The pressure is monitored at one of the chamber ports whereas a turbomolecular pump is attached to a different port. Consequently, the measured base pressure is largely determined by the limited conductance through the chamber center which is almost completely obstructed by the sample holder (chamber ID 0.8 inches, sample holder OD 0.7 inches). Since all components inside the chamber (sample holder, 
diode mounts) are UHV compatible, it should be possible to improve the base pressure significantly by adding pumping to the individual ports of the chamber cross.

The vacuum chamber is supported in part from the stand supporting the entire magnet and in part from the yoke. A photograph of the view inside the magnet yoke with vacuum chamber and seven magnet poles installed is shown in Figure 1(b). The fully assembled system with water cooling and power leads connected is shown in Figure 1(c).

\section{E. Power supplies}

It is possible to use a single bipolar power supply and three high current switches to obtain magnetic fields along the three principle directions, since for these field orientations the currents through all coil pairs can be of the same magnitude. However, since the main design goal of the system is to be able to orient the magnetic field vector in any direction, four independent power supplies are used. We chose unipolar Sorensen DHP 80-125 power supplies (120 A, $80 \mathrm{~V}$ ), and designed four custom FET H-bridge current switching chassis to be able to reverse the currents. 2-A-constant current shunts were built in-house to improve the performance at low currents. The supplies allow incrementing the current in $0.033 \mathrm{~A}$ steps, corresponding to $\sim 180 \mu \mathrm{T}$ field increments along the principle directions with steel poles, or $\sim 24 \mu \mathrm{T}$ with Al poles when only two of the four dipoles are contributing to the field.

\section{F. Sample holders}


The main constraint for the sample holder design was the limitation of its diameter by the access port size to less than 0.8 inches. One of the three available sample holders is shown in Figure 2. It was designed to accommodate a single sample allowing azimuthal rotation. Sample temperatures between $15 \mathrm{~K}$ and $450 \mathrm{~K}$ are possible. The sample is surrounded by a gold mesh mounted on a cylindrical $\mathrm{Cu}$ support. This cage is insulated from other parts of the sample holder and kept at a positive bias voltage. It serves as a collector for electrons photo-emitted from the sample. The electrical insulation between the cryostat and the $\mathrm{Cu}$ disk holding the sample is accomplished by pieces of sapphire that also serve as bearings. Samples are attached typically to the holder using double sided adhesive conductive Carbon tape (SPI Supplies) or small clips as shown in Figure 2. Two additional sample holders accommodating multiple samples and allowing transmission, sample drain current, and total fluorescence yield measurements at temperatures from $15 \mathrm{~K}$ to $450 \mathrm{~K}$ were also developed.

\section{PERFORMANCE}

Important aspects of the magnet performance are the precision with which a desired field vector can be determined by adjusting the current distribution and the ability to measure high precision, i.e. low noise, XMD spectra and loops.

\section{A. Field current relation}

The magnetic performance of the device was characterized using a three axis Hall transducer (3R100-3-2 by Sentron, Switzerland). Figure 3 shows dependence of the 
magnetic field at the magnet center from the current through each of the four dipole pairs. The field vector is oriented along the principle direction $\mathbf{x}, \mathbf{y}$, and $\mathbf{z}$, i.e. the current is of the same magnitude for all four coil pairs. For aluminum poles the current-field relation is linear with a slope of $1.47 \mathrm{mT} / \mathrm{A}$. For steel poles the $B(I)$ curve is very well described by a linear approximation up to $50 \mathrm{~A}$ (deviation is less than $0.5 \%, B / I=10.77 \mathrm{mT} / \mathrm{A}$ ). For further increased current the pole tips start to saturate and the slope of the $B(I)$ curve is reduced towards $1.47 \mathrm{mT} / \mathrm{A}$, the slope for a pole material with relative permeability 1 . The remanent field at zero-current is always less than $0.5 \%$ of the maximum field for a hysteresis loop measured with steel poles but depends on recent current distributions used in the system. The deviation between magnetic fields resulting from the same current distribution in subsequent measurements of loops extending over the same field range is $\leq 0.05 \mathrm{mT}$. Consequently, the remanent field at zero-current can be determined with this precision by a reference measurements using a Hall transducer or a previously thoroughly characterized sample when the exact knowledge of the remanent field is critical, e.g. when an exchange biased system exhibiting significant coercivity but small exchange bias fields is studied.

A comparison of the experimental data (open circles) with the calculated $B(I)$ relationship for steel poles (solid lines) using the software package TOSCA is shown in Figure 3. The experimental values are about $1.5 \%$ smaller than predicted by the model for the entire current range. This deviation is in part a consequence of experimental uncertainties, e.g. in determining the absolute current and in the calibration and positioning of the Hall transducer. The accuracy of the model calculations is limited by the imperfect knowledge of number and location of coil turns and the dependence of the steel 
permeability on temperature and field. The quality of the three dimensional grid used in the finite element calculation is also relevant. Overall, the agreement of model and experiment is remarkably good.

Figure 4 shows a comparison of the calculated and measured spatial variation of the magnetic fields parallel and perpendicular to the field direction for $B$ along $\mathbf{x}, \mathbf{y}, \mathbf{z}$ and again very good agreement is found. Note that the field roll-off with distance from the magnet center point is significantly smaller in the case of $\mathrm{Al}$ poles than in the case of the steel poles.

The excellent agreement of model calculations and experimental results indicate that the precise current distribution for any arbitrary field vector can be obtained using TOSCA. However, to calculate individually the currents for the large number of different field vectors used in many XMD experiments is not practical. An alternative is to estimate the $B(I)$ curves for any current distribution by summing the dipole fields generated by the four dipole pairs individually (Figure 5). A comparison of the experimental $B(I)$ curve (solid line) with a linear fit (dotted line) and the summation of the dipole fields (dashed line) for field vectors along the principle magnet directions shows that the linear approximation provides a significantly better description of the experimental data. The reason is that as a single dipole pair is energized, the pole tip eventually becomes saturated and an increasing fraction of flux passes through unsaturated neighboring poles. When all poles are energized equally, e.g. for fields along the principle directions, this does not occur. In essence the nonlinearity in $B(I)$ for a single pole is enhanced by the existence of unsaturated pole neighbors. 
For coil currents up to $50 \mathrm{~A}$ the current field relation is sufficiently linear to justify the use of a linear model to determine the currents for arbitrary field angle. A more formal description of this model in matrix form gives the current as

$$
H=A I \text { with } A=\alpha\left(\begin{array}{rrrr}
1 & 1 & 1 & 1 \\
1 & -1 & -1 & 1 \\
-1 & -1 & 1 & 1
\end{array}\right)\left(\begin{array}{l}
I_{1} \\
I_{2} \\
I_{3} \\
I_{4}
\end{array}\right)
$$

The proportionality factor $\alpha$ is $\alpha=2.69 \mathrm{mT} / \mathrm{A}$ for steel poles and $\alpha=0.368 \mathrm{mT} / \mathrm{A}$ for Al poles. The signs of the matrix elements depend on the current direction and numbering convention of the poles with respect to the user reference frame.

The current-field relation (1) is not unique, i.e. different current distributions can lead to the same field vector. The extra degree of freedom available when defining the four currents $I_{1}, I_{2}, I_{3}, I_{4}$ to provide a field vector $\left(B_{\mathrm{x}}, B_{\mathrm{y}}, B_{\mathrm{z}}\right)$ can be used to minimize the peak current required. The resulting algorithm, yielding a unique current distribution solution, is defined by:

1. Determine a solution $I_{i}^{0}$ by superposition of the principal-coordinate solutions (defined by the rows of $A$ ).

2. Isolate the largest amplitude current component in $I^{0}: k=\left\{j ;\left|I_{j}^{0}\right| \geq\left|I_{i}^{0}\right|, i=1, \ldots, 4\right\}$, and determine the (square) submatrix $A_{i j}^{k}$ obtained by eliminating the column $k$.

3. Generate a (unique) seconday solution $I^{1}=\left[A^{k}\right]^{-1} B$ 
4. Isolate the largest amplitude current component in the secondary solution $I^{1}: m=\left\{j ;\left|I_{j}^{1}\right| \geq\left|I_{i}^{1}\right|, i=1, \ldots, 4, i \neq k\right\}$

5. The final current distribution is defined such that the amplitudes of the two largest currents in the final configuration are equal: $I^{f}=\alpha I^{0}+(1-\alpha) I^{1}$, where $\alpha=\frac{\left|I_{m}^{1}\right|}{\left(\left|I_{k}^{0}\right|-\left|I_{m}^{0}\right|+\left|I_{m}^{1}\right|\right)}$.

The coil currents are limited to about $100 \mathrm{~A}$ by the ability of the water cooling to remove power deposited in the system. Consequently, the optimization of the current distribution as described above generally increases the accessible field range by reducing the peak current for a specified field direction. Figure 6 shows the ratio of the maximum field obtainable using the linear model, $B_{\max }^{\text {linear model }}$, and the optimized model, $B_{\max }^{\text {optimized }}$, for the same peak current for selected field orientations. $\theta$ is the angle between the field vector and the $\mathbf{x y}$ plane and $\phi$ the angle relative to the $\mathbf{x}$ axis within the $\mathbf{x y}$ plane. The impact of the current optimization is most significant for field vectors along the direction of a magnet dipole pair, i.e. $\theta=35.7^{\circ}$ and $\phi=45^{\circ}$. Using the linear model, the current for the dipole pair along the field direction is about three times as large as the currents in the other three dipoles. Using the current-minimization algorithm all currents are of equal magnitude. The optimization leads to an increase in accessible field range by $50 \%$ along this direction. Field vectors in the principle directions (where all coil currents are equal using the linear model) and within the $\mathbf{x y}, \mathbf{x z}$, and $\mathbf{y z}$ planes (where at least two currents are of the same magnitude in the linear model) do not benefit from current distribution optimization, since the two approaches yield identical distributions. 
To account for the non-linearity of the $B(I)$ relationship neglecting the pole-to-pole interaction, the matrix coefficients become current-dependent of the form $A_{i j}=A_{i j}(I)$.

The simplest approximation in this case is to assume $A_{i j}=A_{i j}\left(I_{j}\right)$, i.e. the nonlinearity due to the saturation of a pole is unaffected by the state of the other coils. The experimental data (Figure 5) demonstrates that a more sophisticated model is needed. Taking the pole-to-pole interaction into account, i.e. the effect of the field generated by one pole on the other poles, leads to matrix coefficients depending on all four currents $A_{i j}$ $=A_{i j}\left(I_{1}, I_{2}, I_{3}, I_{4}\right)$.

\section{B. Spectroscopy results}

The magnet is designed to accommodate transmission experiments, total electron yield measurements by monitoring the sample drain current, and total fluorescence yield measurements with a diode installed at $90^{\circ}$ relative to the $\mathrm{x}$-ray beam.

Figure 7 shows $\mathrm{x}$-ray absorption (XA), XMCD and XMLD spectra measured at the $\mathrm{Tb}$ and Dy $\mathrm{M}_{5,4}$ edges of a $40 \mathrm{~nm}$ Terfenol $\left(\mathrm{Fe}_{66} \mathrm{~Tb}_{9.5} \mathrm{Dy}_{24.0}\right)$ film in transmission geometry. All spectra were obtained at $\mathrm{T}=298 \mathrm{~K}$ and in normal incidence. The XMCD asymmetry signal represents the difference of spectra taken with elliptically polarized x-rays and opposite magnetic fields of $\pm 0.2 \mathrm{~T}$ normalized to their sum. XMLD spectra are obtained with linearly polarized x-rays by applying external fields parallel and perpendicular to the polarization direction. The XMLD asymmetry is defined as difference of those two spectra divided by their sum. The noise of the dichroism signal in both cases is about $10^{-4}$. 
XA spectra, XMCD spectra, and element-specific hysteresis loops of a trilayer sample of 5 monolayers (ML) Co/ $10 \mathrm{ML} \mathrm{Fe} \mathrm{/} 18 \mathrm{ML}$ Ni grown on a $\mathrm{Cu}(100)$ substrate obtained in total electron yield mode are displayed in Figure 8. The noise in the XMCD asymmetry is again about $10^{-4}$. The hysteresis loops are obtained by monitoring the field dependence of the $\mathrm{Fe}, \mathrm{Co}$, and $\mathrm{Ni}_{\mathrm{L}} \mathrm{XMCD}$ signal for external fields at $30^{\circ}$ to the sample surface and x-rays in normal incidence (Figure $7(\mathrm{c})$ ) and $30^{\circ}$ grazing incidence (Figure 7(d)). The Ni magnetization has an easy axis out of plane while the Co easy axis is in the plane of the sample. The coupling of $\mathrm{Ni}$ and Co through the intermediate $10 \mathrm{ML} F e$ layer is antiferromagnetic leading to a rather complex magnetization reversal for magnetic fields applied in the sample plane.

Figure 9 shows $\mathrm{Fe} \mathrm{L}_{3,2}$ total fluorescence yield data of a $\mathrm{CoFe}_{2} \mathrm{O}_{4}$ sample measured at room temperature and an angle of $\mathrm{x}$-ray incidence of $5^{\circ}$ relative to the sample surface. The detection diode was installed at $90^{\circ}$ to the incoming x-ray beam. The fluorescence data is clearly influenced by saturation effects at the $\mathrm{L}_{3}$ as expected for any concentrated sample. An XMCD asymmetry of $0.5 \%$ is observed at the $\mathrm{Fe}_{3}$ edge although the signal to noise on the spectrum is significantly worse than for electron yield and transmission signal due to the comparatively much smaller fluorescence signal

The spectroscopy results show clearly that the setup is very well suited for the measurement of precision XA, XMCD, and XMLD spectra as well as element-specific hysteresis loops. A combination of XMCD spectroscopy on ferromagnetic Fe and XMLD measurements on antiferromagnetic $\mathrm{NiO}$ performed with our system has recently be used to study the formation of an antiferromagnetic exchange spring in a ferromagnetantiferromagnet bilayer. [19] 


\section{OUTLOOK}

External fields of about $5 \mathrm{~T}$ are needed to align the magnetic moments along the hard magnetization direction in many intriguing magnet materials like molecular magnets [20], magnetic nanocrystals [21], functional magnetic oxides [22], and ferromagnetic semiconductors [23]. Fields of this magnitude can only be achieved with a superconducting device. We are currently developing the basic technology for a superconducting multiple-pole magnet that provides magnetic fields of up to $5 \mathrm{~T}$ in any direction, allows full field reversal in a few seconds, sufficient access for a variety of detectors as well as flexible sample detector geometries suitable for scattering experiments. The new capabilities amount to an improvement in peak operating field by $50 \%$ and in switching rate by a factor of more than 500 compared to existing superconducting devices [16]. Rapid field ramping suitable for point-by-point field reversal in spectroscopy experiments is essential to achieve the sensitivity needed to study novel magnetic nanostructures.

The key technical difficulties arise from the combined requirements of high field, sufficient sample and detector access areas, rapid field variation, and the provision of a scattering plane with its accompanying radiation heat load. The main and new aspects of this research and development project are the following:

- High switching rates can induce magnet quenches from heating associated with superconductor AC losses, i.e. hysteresis (losses associated with changing flux penetration into the superconductor), coupling currents (essentially eddy currents linking different superconducting filaments in the superconducting strand), and eddy 
currents in neighboring materials. These can be minimized by proper selection of conductor (minimal filament size, appropriate filament twist pitch, and tolerance to local temperature excursions by appropriately adjusting the residual resistance ratio (RRR) of the copper stabilizer), appropriate selection and design of components, and optimization of the cryogenic design.

- It should be possible to achieve peak fields of $5 \mathrm{~T}$ by using state of the art $\mathrm{Nb}_{3} \mathrm{Sn}$ superconductors stemming from the Conductor Development Program within LBNL's Supercon group [24]. $\mathrm{Nb}_{3} \mathrm{Sn}$ has substantially higher critical current than $\mathrm{NbTi}$, and has a much higher critical temperature ( $20 \mathrm{~K}$ versus $9.8 \mathrm{~K})$, making it far more tolerant of temperature variations. In particular, recent progress in RRP (restacked-rod processed) superconductor has resulted in critical currents $\left(J_{\mathrm{c}}\right)$ as high as $8900 \mathrm{~A} / \mathrm{mm}^{2}$ at $6 \mathrm{~T}$ and $4.2 \mathrm{~K}$, a $50 \%$ increase in $J_{\mathrm{c}}$ in $\mathrm{Nb}_{3} \mathrm{Sn}$ within about 3 years, and $300 \%$ higher than can be obtained with $\mathrm{NbTi}$ superconductors.

Research to address these questions and develop a high field eight-pole magnet is in progress in our laboratory.

\section{Acknowledgements}

The design and construction of the eight pole electromagnet was the work of many people. We want to acknowledge the support by J. Acre, B.J. Bailey, M.P. Fahmie, W.J. Gath, A. Pekedis, Z. Hussain, Y.U. Idzerda, R.S. Mueller, J.S. Pepper, R.D. Schlueter, J. Spring, J. Turner, and A.T. Young. Samples were kindly provided by Peter Fischer (LBNL) and Z.Q. Qiu (UC Berkeley). The Advanced Light Source is supported by the Director, Office of Basic Energy Sciences, of the U.S. Department of Energy. 


\section{References}

[1] B. T. Thole, G. van der Laan, and G. A. Sawatzky, Phys. Rev. Lett. 55, 2086 (1985).

[2] G. van der Laan, B. T. Thole, G. A. Sawatzky, J. B. Goedkoop, J. C. Fuggle, J.-M. Esteva, R. Karnatak, J. P. Remeika, and H. A. Dabkowska, Phys. Rev. B 34, 6529 (1986).

[3] G. Schütz, W. Wagner, W. Wilhelm, P. Kienle, R. Zeller, R. Frahm, and G. Materlik, Phys. Rev. Lett. 58, 737 (1987).

[4] C. T. Chen, F. Sette, Y. Ma, and S. Modesti, Phys. Rev. B 42, 7262 (1990).

[5] Y. U. Idzerda, L. H. Tjeng, H.-J. Lin, C. J. Gutierrez, G. Meigs, and C.T. Chen, Phys. Rev. B 48, 4144 (1993).

[6] P. Morrall, F. Schedin, G. S. Case, M. F. Thomas, E. Dudzik, G. van der Laan, and G. Thornton, Phys. Rev. B 67, 214408 (2003).

[7] M. G. Samant, J. Stöhr, S. S. P. Parkin, G. A. Held, B. D. Hermsmeier, F. Herman, M. Van Schilfgaarde, L.-C. Duda, D. C. Mancini, N. Wassdahl, and R. Nakajima, Phys. Rev. Lett. 72, 1112 (1994).

[8] B. T. Thole, P. Carra, F. Sette, and G. van der Laan, Phys. Rev. Lett. 68, 1943 (1992).

[9] P. Carra, B. T. Thole, M. Altarelli, and X. Wang, Phys. Rev. Lett. 70, 694 (1993).

[10] C. T. Chen, Y. U. Idzerda, H.-J. Lin, N. V. Smith, G. Meigs, E. Chaban, G. H. Ho, E. Pellegrin, and F. Sette, Phys. Rev. Lett. 75, 152 (1995).

[11] G. van der Laan, Phys. Rev. Lett. 82, 640 (1999). 
[12] S. S. Dhesi, G. van der Laan, E. Dudzik, and A. B. Shick, Phys. Rev. Lett. 87, $67201(2001)$.

[13] V. Chakarian, Y. U. Idzerda, G. Meigs, E. E. Chaban, J.-H. Park, and C. T. Chen, Appl. Phys. Lett. 66, 3368 (1995).

[14] E. Dudzik, S. S. Dhesi, and G. van der Laan, SRN 13, 18 (2000).

[15] C. S. Hwang, C. T. Chen, P. C. Chang, H. H. Chen, C. H. Chang, and M. H. Huang, J. Magn. Magn. Mater. 209, 169 (2000).

[16] C. S. Hwang, C. T. Chen, C. H. Chang, C. Y. Liu, F. Y. Lin, B. Wang, and R. Wahrer, J. Magn. Magn. Mater. 239, 586 (2002).

[17] A.T. Young, J. Feng, E. Arenholz, H.A. Padmore, T. Henderson, S. Marks, E. Hoyer, R. Schlueter, J.B. Kortright, V. Martynov, C. Steier, andG. Portmann, Nucl. Instr. and Meth. A 467-468, 549 (2001).

[18] We used LORD® CASTALL E-301 epoxy resin with RT-1 room temperature cure hardener in this process.

[19] A. Scholl, M. Liberati, E. Arenholz, H. Ohldag, and J. Stöhr, Phys. Rev. Lett. 92, 247201 (2004).

[20] L. Thomas, F. Lionti, R. Ballou, D. Gatteschi, R. Sessoli, and B. Barbara, Nature 383, 145 (1996).

[21] S. Sun, C. B. Murray, D. Weller, L. Folks, and A. Moser, Science 287, 1989 (2001). 
[22] H. Zheng, J. Wang, S. E. Lofland, Z. Ma, L. Mohaddes-Ardabili, T. Zhao, L. Salamanca-Riba, S. R. Shinde, S. B. Ogale, F. Bai, D. Viehland, Y. Jia, D. G. Schlom, M. Wuttig, A. Roytburd, and R. Ramesh, Science 303, 661 (2004).

[23] H. Ohno, Science 281, 951 (1998).

[24] R. M. Scanlan and D. R. Dietderich, IEEE Trans. Appl. Supercond. 13, 1536 (2003). 


\section{Figure Captions}

\section{Figure 1}

Photographs of the eight pole resistive magnet and its components. (a) One of the eight coil pack/pole units. (b) View inside the magnet yoke with vacuum chamber and seven magnet poles installed. (c) Fully assembled system with water cooling and power leads connected.

\section{Figure 2}

(a) Front and (b) back view of a sample holder allowing in-plane rotation of a single sample for temperatures between $15 \mathrm{~K}$ and $450 \mathrm{~K}$.

\section{Figure 3}

Calculated and measured current-field relation for magnetic fields along the principle axes $\mathbf{x}, \mathbf{y}$, and $\mathbf{z}$ of the device. (a) Comparison of the experimental data obtained for steel poles (circles) and Aluminum poles (square) with results of model calculations using TOSCA (solid line) and a linear fit to the experimental data (dashed line), respectively. (b) Ratio of the theoretical and experimental values for steel poles (solid line) and Aluminum poles (dashed line). 


\section{Figure 4}

Calculated and measured spatial variation of the magnetic field for field vectors oriented along the principle axes $\mathbf{x}, \mathbf{y}$, and $\mathbf{z}$ of the device. The experimental data is shown by circles for steel poles and squares for Aluminum poles. The corresponding calculated profiles are shown by solid lines for steel poles and dashed lines for Aluminum poles. The field decrease with increasing distance from the magnet center along the field direction and the field increase with increasing distance perpendicular to the field orientation are in excellent agreement with the model calculations.

\section{Figure 5}

Comparison of the measured field-current dependence (solid line) with a linear fit of the data up to $50 \mathrm{~A}$ (dotted line) and an estimated relation obtained by summing the dipole fields generated by the four dipole pairs individually (dashed line). The field-current dependence for a single pole is indicated by a dash-dotted line.

\section{Figure 6}

Ratio of the maximum field obtainable using the optimized model, and $B_{\max }^{\text {optimized }}$, and the

linear model, $B_{\max }^{\text {linear model }}$, for the same peak current. $\theta$ is the angle between the field vector and the $\mathbf{x y}$ plane and $\phi$ the angle relative to the $\mathbf{x}$ axis within the $\mathbf{x y}$ plane. The impact of the current optimization is most significant for field vectors along the direction of a dipole pair, i.e. $\theta=35.7^{\circ}$ and $\phi=45^{\circ}$. 


\section{Figure 7}

$\mathrm{Tb}$ and $\mathrm{Dy} \mathrm{M}_{5,4}$ spectra and XMD data measured at room temperature and in normal incidence from a $40 \mathrm{~nm}$ Terfenol $\left(\mathrm{Fe}_{2} \mathrm{~Tb}_{0.3} \mathrm{Dy}_{0.7}\right)$ sample in transmission geometry. (a) XA spectra. (b) XMCD asymmetry measured with elliptically polarized radiation and point-by-point reversal of a 0.2 T-field oriented along the sample normal. (c) XMLD asymmetry measured with linearly polarized x-rays and point-by-point reversal of the magnetic field with the sample plane, i.e. the difference of spectra obtained with a $0.2 \mathrm{~T}$ field oriented parallel and perpendicular to the polarization direction normalized to their sum is shown.

\section{Figure 8}

XA spectrum, XMCD spectrum, and element specific hystersis loops for a trilayer sample of 5 monolayers (ML) Co/ $10 \mathrm{ML} \mathrm{Fe} \mathrm{/} 18 \mathrm{ML} \mathrm{Ni}$ on a $\mathrm{Cu}(100)$ substrate. (a) XA data . (b) XMCD asymmetry spectrum measured with elliptically polarized radiation and pointby-point reversal of the magnetic field of $0.2 \mathrm{~T}$. (c) Element-specific loops with the magnetic field parallel to the $\mathrm{x}$-ray beam, i.e. at $30^{\circ}$ relative to the sample surface. The $\mathrm{Ni}$ magnetization has an easy axis out of plane while the Co easy axis is in the plane of the sample. The magnetic coupling of $\mathrm{Ni}$ and $\mathrm{Co}$ through the intermediate $10 \mathrm{ML}$ Fe layer is antiferromagnetic leading to a complex magnetization reversal for magnetic fields

applied in the sample plane. (d) Element-specific loops measured at normal incidence 
with the magnetic field at $30^{\circ}$ relative to the sample surface, representing the out-of-plane component of the moments.

\section{Figure 9}

$\mathrm{Fe} \mathrm{L}_{3,2}$ total fluorescence yield data of a $\mathrm{CoFe}_{2} \mathrm{O}_{4}$ sample measured at room temperature at an angle of x-ray incidence of $5^{\circ}$ to the sample normal with a diode at $5^{\circ}$ to the sample surface. (a) The fluorescence spectrum measured with linear polarized radiation is clearly influenced by saturation effects as expected for concentrated samples. (b) The XMCD asymmetry was obtained with external fields of $\pm 0.5 \mathrm{~T}$ applied along the $\mathrm{x}$-ray beam. 


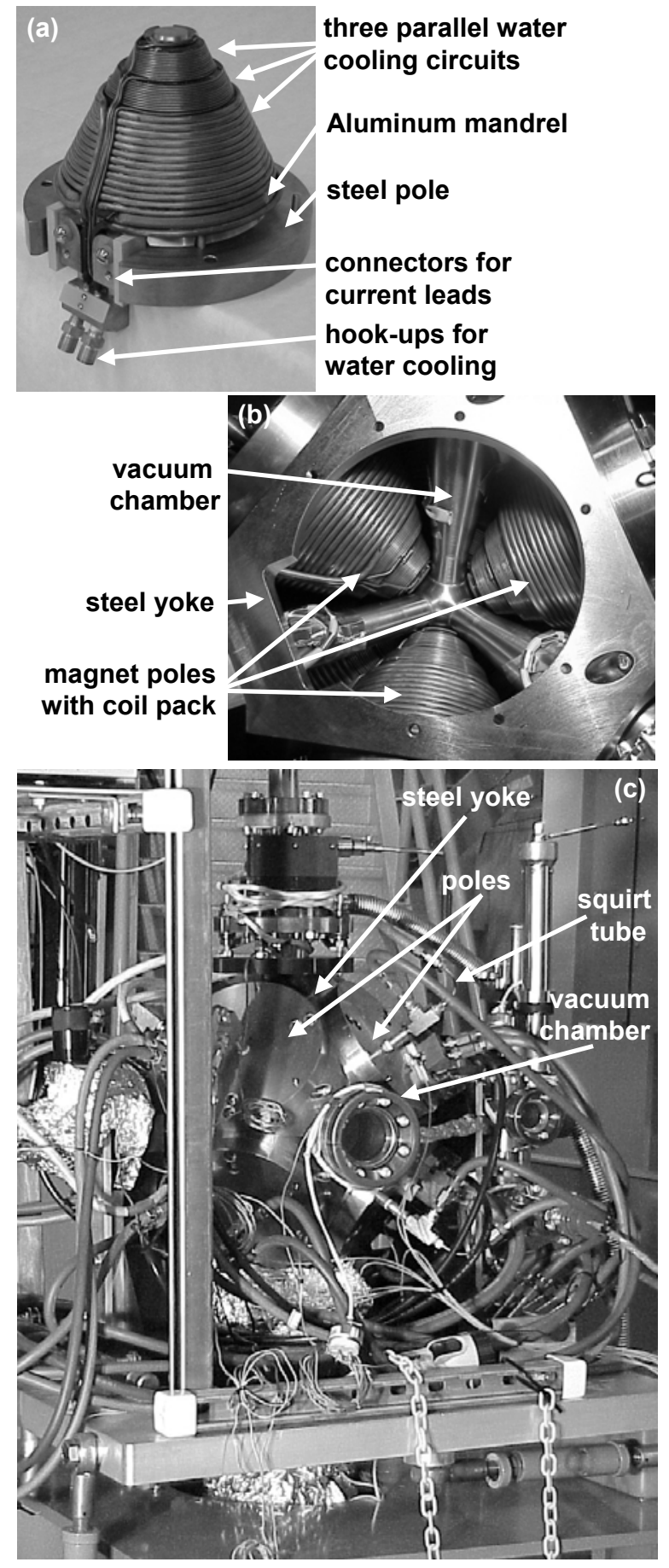

Figure 1 


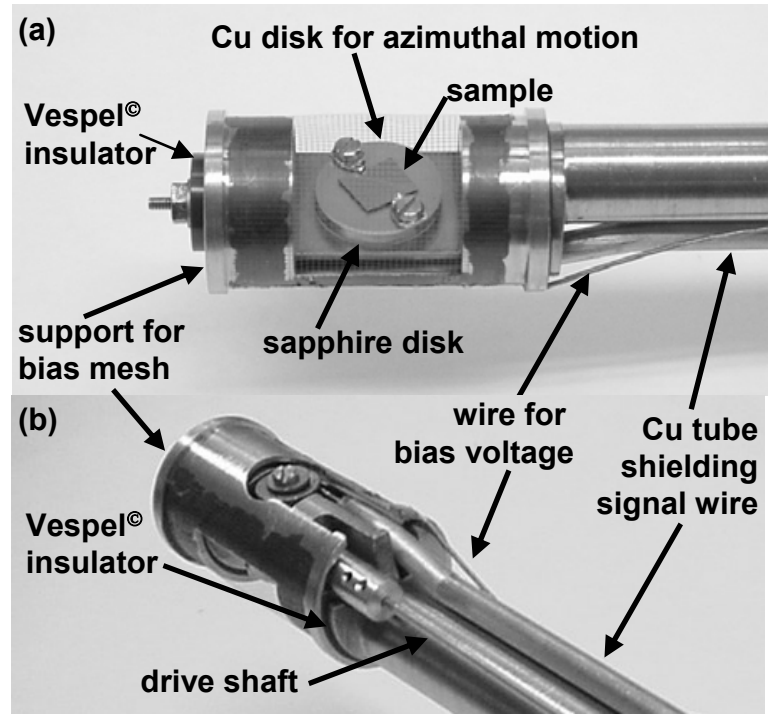

Figure 2 


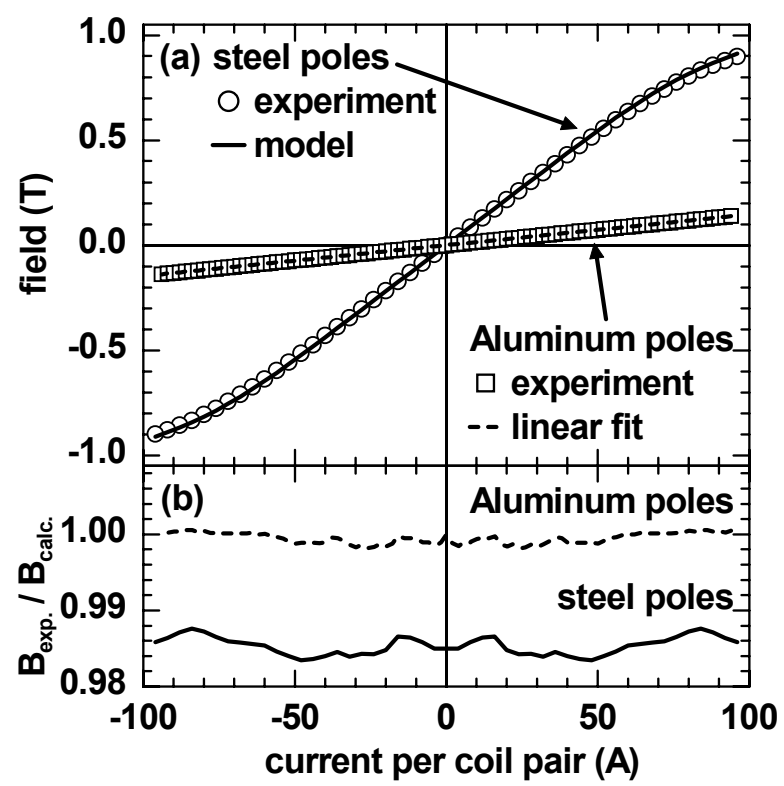

Figure 3 


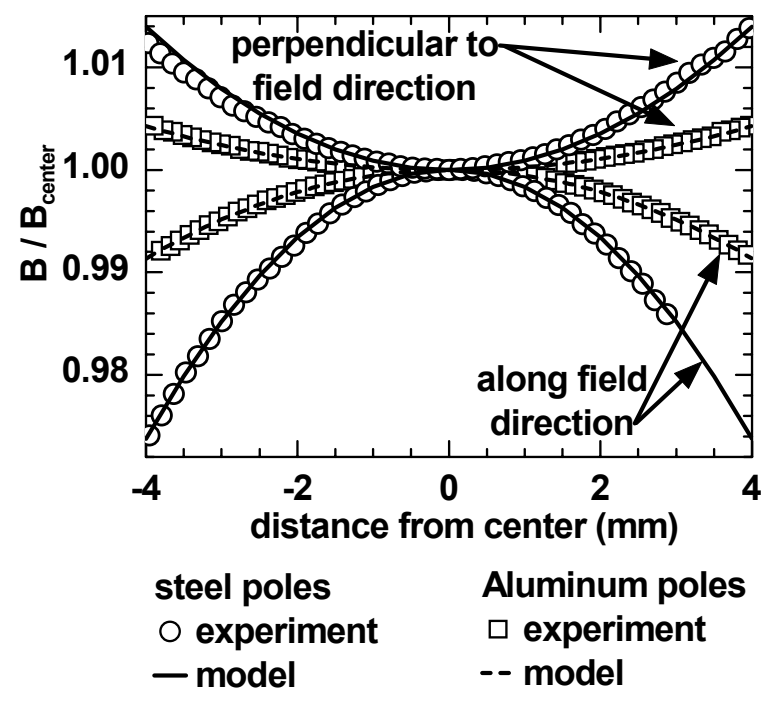

Figure 4 


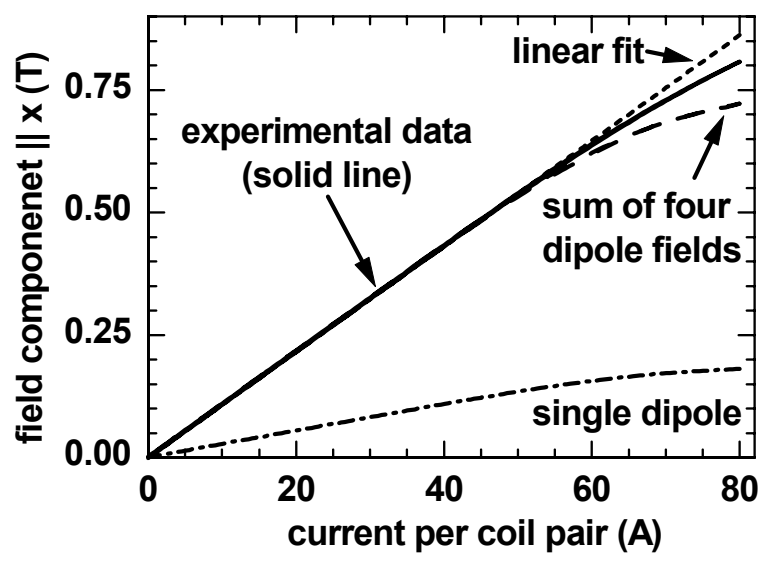

Figure 5 


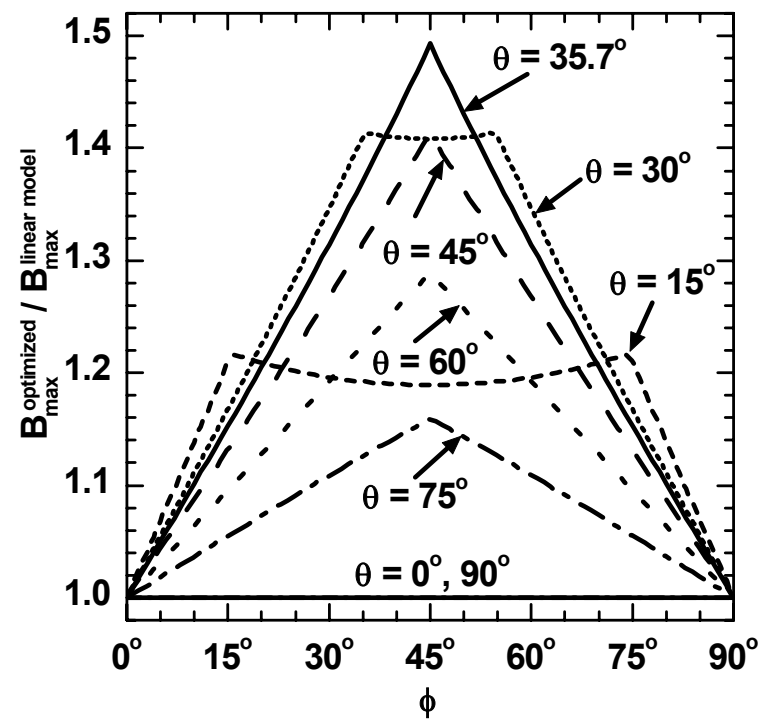

Figure 6 


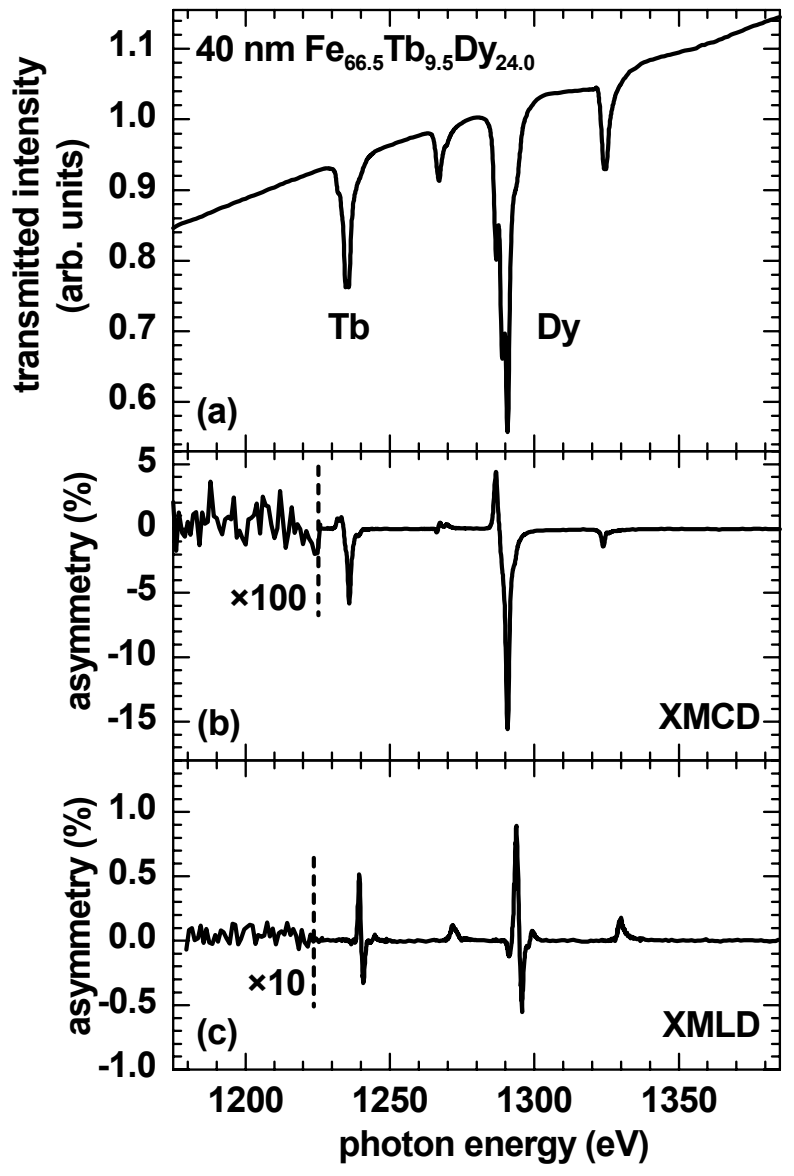

Figure 7 

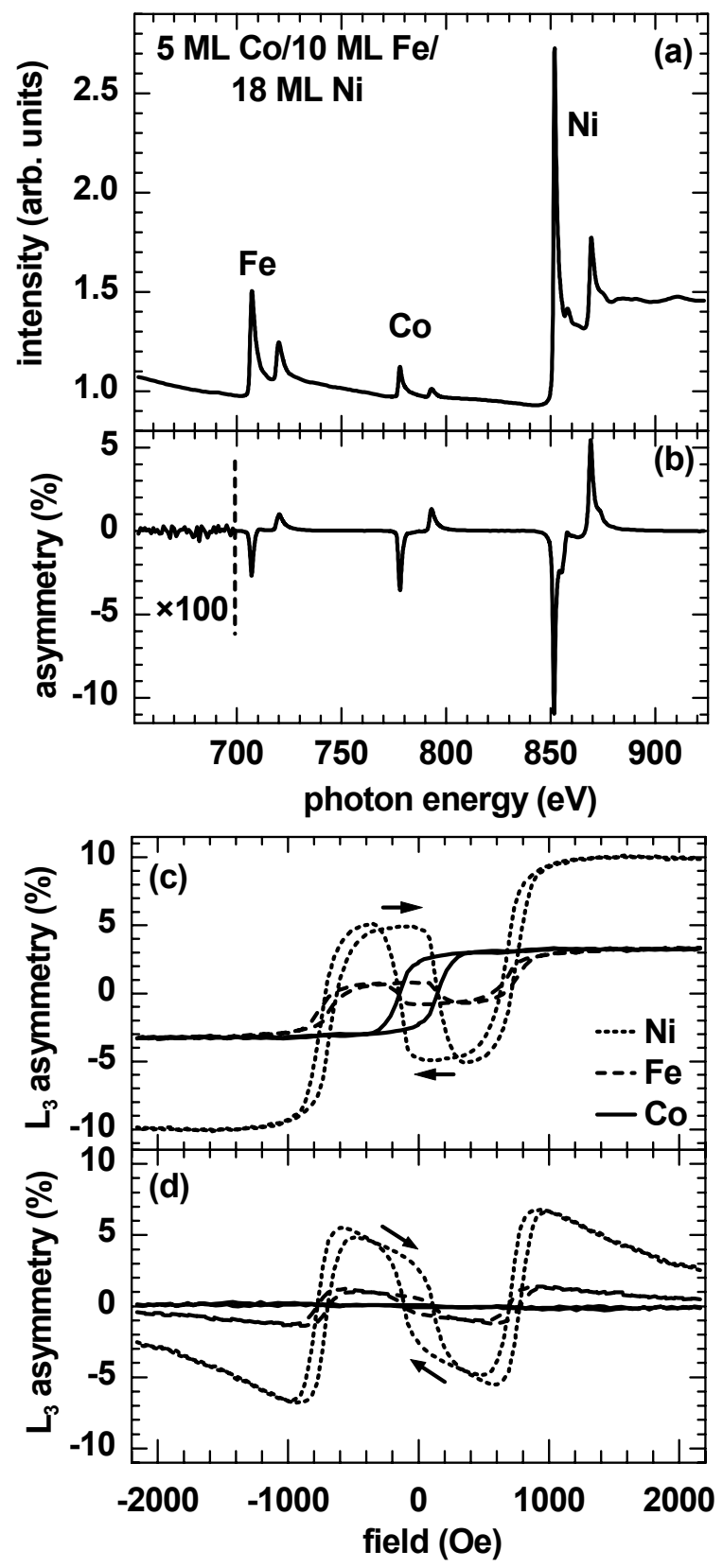

Figure 8 


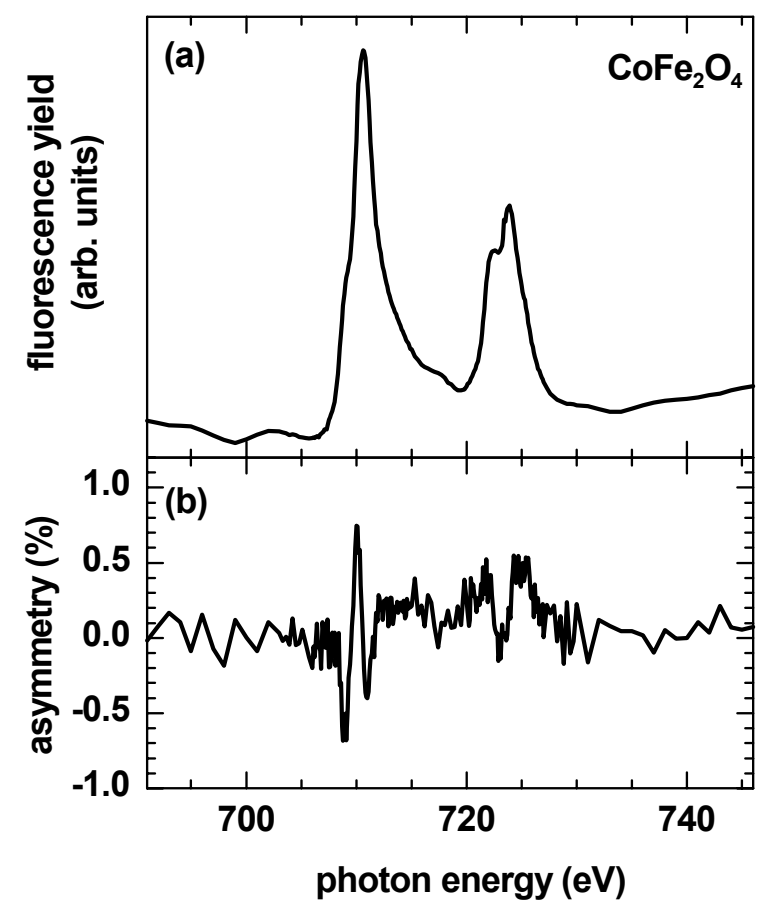

Figure 9 